\title{
The Application of Mixed Teaching Mode Based on SPOC and Online Judge in Data Structure Course
}

\author{
Ding Linhua ${ }^{1, a,{ }^{*}}$, Wang Xiaojie ${ }^{1, b}$, Wang Jiuru ${ }^{1, c}$ and Liu Li ${ }^{1, d}$ \\ ${ }^{1}$ School of Information Science and Engineering, Linyi University, Linyi, ShanDong, China

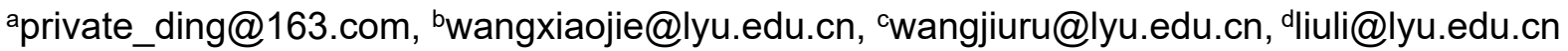 \\ *Ding Linhua
}

Keywords: Online Judge, SPOC, Data Structure.

\begin{abstract}
SPOC provides personalized learning. Online evaluation is an experimental platform for programming courses. Rain Classroom is a teaching information tool based on WeChat. The mixed teaching mode combines the three elements into the data structure course to achieve in class and extracurricular, online and offline, mobile and fixed, and personalized and diversified teaching.
\end{abstract}

\section{基于SPOC和在线评测的混合教学模式在数据结构课程中的应用}

\author{
丁林花 ${ }^{1, a}$ ，王晓洁1,b, ${ }^{*}$, 王九如 ${ }^{1, c}$, 刘丽1,d \\ 1临沂大学信息科学与工程学院, 临沂, 山东, 中国
}

aprivate_ding@163.com, bwangxiaojie@lyu.edu.cn, `wangjiuru@lyu.edu.cn, dliuli@lyu.edu.cn

*丁林花

关键词: 在线评测;SPOC; 数据结构

中文摘要. SPOC提供个性化学习, 在线评测是程序设计类课程的实验平台, 雨课堂是基于微 信的教学信息化工具, SPOC+PTA+雨课堂的混合教学模式将三者有机结合应用到数据结构课 程中，实现课内课外、线上线下、移动固定的个性化、多样化教学。

1. 引言

《数据结构》是计算机科学中一门重要的专业基础课。通过该课程的学习, 不仅使学生 深刻地理解数据结构的逻辑结构和物理结构的基本概念以及有关算法, 培养学生的程序设计 能力, 更重要的是培养学生的自主学习能力、工程应用能力和创新能力。长期的教学实践发 现, 学习该课程时, 学生的程序设计语言基础参差不齐, 小规模的在线课程SPOC为个性化教 学提供了可能。同时程序设计能力的提高和实际解决问题的能力培养则需要大量的编程训练, 在线评测系统作为SPOC课程的有效补充和实验平台可以取得非常好的效果。雨课堂是清华大 学联合学堂在线共同研发的新型教学工具, 利用微信这一受学生欢迎的流行的移动互联网社 交工具, 雨课堂可以灵活地开展混合式教学。将雨课堂和在线评测引入到数据结构SPOC课程 中, 如何将这三种教学模式有效的组织起来, 既能让不同层次学生喜爱, 还能够让学生能力 从知识、应用到创新呈阶梯型递进是一个值得研究的课题。 


\section{SPOC+PTA+雨课堂的混合教学模式}

SPOC主要为学生提供视频资源, 在线评测主要提供大量编程实践, 雨课堂主要提供随堂 测试和讨论, 这种 “课内课外、线上线下、移动固定” 互融互补的多样化混合式教学模式让 不同层次的学生都必须学习、爱上学习、自主学习。

\subsection{SPOC课程资源和在线评测系统选取}

MOOC资源的选择需结合学生的实际情况和不同需求, 既要为能力高的学生提供更好更 高的学习资源，更要兼顾基础一般或不好的学生。基于这两点考虑，作如下选择：

选取中国大学MOOC平台浙江大学《数据结构》建立异步SPOC课程。该课程既有基础知 识篇, 还有小白专场, 还有适合高水平学生的习题、操作选讲, 能够兼顾到各个层次的不同 学生需求。

选用浙江大学程序设计类实验辅助平台PTA作为在线评测平台。该平台既可以完成编程 练习, 也可以完成测验和考试。更重要的是该平台不同于传统的在线评测系统要么通过得满 分要么通不过得零分, PTA平台是根据测试点评测的, 每个测试点都有单独的得分。这样的 评测方式即可以增强学生的兴趣和自信心，还可以通过不正确的测试用例检查程序的逻辑漏 洞和完备性，进而一步步地提高程序设计能力。

\section{2 线上线下、课上课外学习内容设计}

线上线下的混合教学模式需要重新安排线上线下的教学环节，尤其是线上线下、课上课 外的自学内容、课堂讲授内容切分必须明确, 并能够及时通过配套评测手段检验学习效果。

以本课程第一章 算法部分为例，学习内容设计如表1所示。该表中明确的规定了哪些是 自学内容, 哪些是线下学习和讨论内容, 以及每一部分的学习任务和程度，通过观看视频、 雨课堂推送自学/预习课件等形式学生可以课下完成线上自学任务, 配合雨课堂课前推送试卷、 雨课堂课中随堂测试和课中投稿、分组讨论等翻转课堂形式, 有效地检验学生的学习情况。 同时在PTA上提供为每个层次学生大量编程实践, 提高学生程序设计能力和知识运用能力。

表1 线上线下、课上课外学习内容设计

\begin{tabular}{|c|c|c|c|c|}
\hline 项目 & 学习形式 & 学习任务 & 学习方式 & 成绩评测方式 \\
\hline 算法定义 & 线上自学 & 了解算法定义 & $\begin{array}{c}\text { 观看SPOC视频: } \\
\text { 算法的定义 }\end{array}$ & 雨课堂测试 \\
\hline 算法评价 & 线上自学 & 掌握好的算法标准 & $\begin{array}{c}\text { 观看SPOC视频: } \\
\text { 什么是好的算法 }\end{array}$ & 雨课堂测试 \\
\hline 算法时间复杂度和 & 线下学习 & 掌握算法时间复杂度和空间 & 课堂讲授、师生 \\
空间复杂度的计算 & 及讨论 & 复杂度的计算方法 & 两课堂讨论、投稿 \\
\hline 泣用实例: 最大子序 & 编程实践 & 转 & 或课堂分组讨实践体会不同时间复杂 \\
列和问题 & 练习 & $\begin{array}{c}\text { 度的算法的效率, 提高优化 } \\
\text { 小组编程实践 }\end{array}$ & PTA在线编程成绩 \\
\hline
\end{tabular}

\section{3 线上线下教学过程}

一、课前自学与测试 
为了使学生明确自学任务, 根据教学进度及时通过雨课堂给学生推送学习任务单如表2 所示。同时雨课堂推送配套课件和自学测试题目。自学测试题目可以放到课件中, 也可以单 独做成一个试卷。

表2 学习任务单

\begin{tabular}{|c|c|c|}
\hline 学习任务单 & 项目 & 具体内容 \\
\hline \multirow{4}{*}{ 算法内容 } & 观看视频 & 观看SPOC视频: 1.2 .1 和1.2.2 \\
\cline { 2 - 3 } & 阅读课件 & 看完雨课堂推送的自学配套课件或测试课件 \\
\cline { 2 - 4 } & 测试与讨论 & 课上完成雨课堂随堂测验和相关讨论 \\
\cline { 2 - 4 } & 在线编程 & 完成PTA编程题目 \\
\hline
\end{tabular}

二、课上教学加雨课堂测试和讨论

线下课堂, 首先对课前测试中得分率低的问题进行点评和讲解。对于教学中的重点难点 则采用教师讲解、雨课堂讨论等多种形式互动学习, 同时使用雨课堂测试考察学习效果。为 了培养学生的独立思考能力和编程思维, 有时也会使用雨课堂分组功能在课堂上进行分组讨 论, 并及时使用投屏功能显示各个小组的独立见解。

三、学生分组讨论和作业

学生按照自由组队方式每4人一个小组, 所有课堂讨论或练习都以小组为单位, 小组表现 计入课堂表现成绩, 兑现平时成绩加分。并且布置四次课下小组大作业, 从设计、实现、测 试到文档编写各个环节考场学生团队协作和应用创新能力。

四、翻转课堂灵活使用各种教学互动方法

在课上授课过程中, 改变以前教师讲解为主的教学模式, 灵活采用各种师生互动模式组 织翻转课堂。比如单链表求倒数第 $\mathrm{K}$ 个元素、循环队列实现中队空队满判定问题、经典递归 问题-汉诺塔, 采用游戏方式让学生体会其实现过程。比如顺序表基本操作、单链表基本操作 则让学生在PTA上分组练习, 展示学生成果, 当场点评。再比如栈和队列的基本性质涉及的 历年考研题目, 让学生分组完成不同题目, 然后派代表课堂讲解, 记录小组课堂表现。

\section{3 学习效果评定}

学生学业成绩全部来源于平时形成的过程性成绩, 包括线上和线下两部分成绩, 其中线 上成绩包括在线评测系统实验成绩、单元测试成绩、期末考试成绩，线下成绩是雨课堂随堂 测验/考试成绩和小组大作业成绩。具体成绩评价体系如表3所示。

表3 学生过程性成绩评价体系

\begin{tabular}{|c|c|c|c|c|}
\hline & 评价项目 & 评价主体 & 评价目标 & 权重 (百分比) \\
\hline \multirow{4}{*}{ 线上 } & SPOC自学成绩 & 雨课堂试卷即时评测 & 知识了解程度和自主效果 & 10 \\
\cline { 2 - 5 } & PTA实验 & 系统即时评测 & 知识应用能力、编程能力 & $\begin{array}{c}\text { 达到 } 80 \% \text { 其它 } \\
\text { 成绩才有效 }\end{array}$ \\
\cline { 2 - 5 } & PTA单元测试 & 系统即时评测 & 知识应用能力、编程能力 & 20 \\
\cline { 2 - 5 } & PTA期末考试 & $\begin{array}{c}\text { 系统即时评测+教师 } \\
\text { 综合考察学生知识掌握程度、 }\end{array}$ & 30 \\
& 评价 & 知识应用能力和程序设计能力 & 30 \\
\hline
\end{tabular}




\begin{tabular}{|c|c|c|c|c|}
\hline \multirow{4}{*}{ 线下 } & 雨课堂随堂测验/小考 & 系统即时评测 & $\begin{array}{c}\text { 基本知识、教学重点、基本技 } \\
\text { 能的掌握情况 }\end{array}$ & 10 \\
\cline { 2 - 5 } & 雨课堂参与讨论 & 教师评价 & $\begin{array}{c}\text { 学习态度、协作精神、自主思 } \\
\text { 考和创新能力 }\end{array}$ & 10 \\
\cline { 2 - 5 } & 小组大作业 & $\begin{array}{c}\text { 教师评价+组长内评+ } \\
\text { 组间互评 }\end{array}$ & $\begin{array}{c}\text { 获取知识能力、知识应用能力、 } \\
\text { 创新能力和团队协作能力 }\end{array}$ & 20 \\
\hline
\end{tabular}

该过程性评价体系采用从低到高的递进式评价层次，从学生知识掌握能力到知识运用能 力再到创新能力都纳入评价体系。

\section{4. 结束语}

对于程序设计类课程, 既需要提供个性化学习、融合在线学习和面对面学习, 还要最大 程度为学生提供实际的编程实践平台。SPOC+PTA+雨课堂的混合式教学模式不但为不同层次 学生提供了丰富的个性化的学习资源, 还利用当前互联网土著一族喜欢的移动工具微信进行 各种课上课下翻转互动, 让学生随时随地都能够自主学习。实践证明, $\mathrm{SPOC}+$ 在线评测 + 雨课 堂的智慧式混合教学模式, 能够更加丰富数据结构课程的资源, 进一步提升教师的教学水平 和教学质量, 大幅度的提高学生的学习兴趣和程序设计能力。

\section{致谢}

本文为临沂大学教学信息化研究项目《SPOC+在线评测+雨课堂的智慧式混合教学模式在 数据结构课程中的研究与应用》、教学研究与改革项目《基于在线评测和项目导向的 VC ++ 应用开发教学模式研究》和学生学习评价改革课程《数据结构》的阶段性成果之一, 同时受 到山东省本科高校教学改革研究项目《地方高校基于校企协同的新工科专业改造升级探索与 实践-以软件工程为例》（M2018X058）的资助。

\section{References}

[1] Dai Chengqiu, Hybrid Teaching Practice and Evaluation in Data Structure Course, Data Structure Course ,vol.1, pp. 80-83, 2019.

[2] Yu Qingmei and Mao LiuLiu, Practical Teaching Model of "Data Structure" in Local Colleges and Universities under Background of MOOCs, Experimental Technology and Management, vol.36, pp. 190-194, 2019.

[3] Chen Yefang and $\mathrm{Hu}$ Wang Xiaoli, Online Practice of Programming Course in Blended Teaching Model, Journal of NingBo University, vol.41.1, pp. 103-107, 2019.

[4] Li Ling and Chen Chao, The Practice of Flipping Classroom Teaching of Science and Technology Information Retrieval Course on Rain Classroom, Library and Information Service, vol. 63.12, pp. 66-71, 2019. 